\title{
FUZZY NORMAL CONGRUENCES AND FUZZY COSET RELATIONS ON GROUPS
}

\author{
S. Khosravi Shoar \\ Department of Mathematics \\ Fasa University \\ Fasa, IRAN
}

\begin{abstract}
The aim of this work is to introduce the concept of a fuzzy normal congruence on a group and illustrate this subject by some examples. We also introduce the concept of a fuzzy coset relation on a group and define a natural composition between two fuzzy coset relations and by this operation we prove that the set of all fuzzy coset relations is a group.
\end{abstract}

AMS Subject Classification: 03E72, 20N25

Key Words: fuzzy relation, fuzzy congruence, fuzzy normal congruence, fuzzy coset relation

\section{Introduction}

L.A. Zadeh [11] introduced the concept of fuzzy set,.and since then there has been a tremendous interest in the subject due to its diverse applications ranging from engineering and computer science to social behavior studies.

This concept is very general and many researchers applied this subject to the various structures and obtained some useful results in their areas. A. Rosenfeld [7] formulated the concept of a fuzzy subgroup and showed how some basic notions of group theory should be extended in an elementary manner to develop the theory of fuzzy groups. J.P. Kim and D.R. Bae [3] characterized the composition of two congruence relations in semigroups and groups. They also defined a fuzzy congruence class and studied this property. N.P. Mukherjee

$\begin{array}{lr}\text { Received: } & \text { October 24, } 2016 \\ \text { Revised: } & \text { April 10, } 2017 \\ \text { Published: } & \text { July 14, } 2017\end{array}$

(C) 2017 Academic Publications, Ltd. url: www.acadpubl.eu 
[5] introduced and studied the concepts of fuzzy normal subgroups and fuzzy cosets in groups. He proved that $\mu$ is a fuzzy normal subgroup of finite group $\mathrm{G}$ if and if the level subgroups of $\mu$ are normal subgroups of $\mathrm{G}$.

In this work, by deep view, we extend the concepts of fuzzy normal subgroups and fuzzy cosets to the fuzzy relation and introduce the concepts of fuzzy normal congruences and fuzzy coset relations in groups. We also define the operation $\odot$ on fuzzy relations and show that the family of all fuzzy congruences with this operation in commutative semigroup is a semigroup and clarify that the set of all fuzzy coset relations obtained from fuzzy normal congruences with operation $\odot$ is a group.

\section{Preliminaries}

Note. Hereinafter, G under operation $*$ is always a group unless otherwise specified.

Definition 2.1. $[2,6]$ Let $X$ be a non-empty set. A fuzzy relation on $X$ is a map $\eta$ from $X \times X$ to the $[0,1]$, and $\mathrm{R}(\mathrm{X})$ will denote the set of all fuzzy relations on $\mathrm{X}$.

Definition 2.2. $[6,9]$ Let $\varphi, \psi \in R(X)$. Then:

(i) $\varphi \subseteq \psi$ if and only if $\forall x, y \in X, \varphi(x, y) \leq \psi(x, y)$.

(ii) $(\varphi \cup \psi)(x, y)=\varphi(x, y) \vee \psi(x, y)$.

(ii) $(\varphi \cap \psi)(x, y)=\varphi(x, y) \wedge \psi(x, y)$.

(iii) $\varphi^{-1}(x, y)=\varphi(y, x)$.

(v) $(\varphi \circ \psi)(x, y)=\bigvee_{z \in X}\{\varphi(x, z) \wedge \psi(z, y)\}$.

Definition 2.3. [6,3] A fuzzy relation $R$ on $\mathrm{X}$ is called a fuzzy equivalence or similarity relation on $\mathrm{X}$ if:

(i) $R(x, x)=1$ for all $x \in X$ (reflexive).

(ii) $R(x, y)=R(y, x)$ for all $x, y \in X$ (symmetric).

(iii) $R \circ R \leq R$ (transitive).

Definition 2.4. [3, 4] Let $\mathrm{G}$ be a semigroup(group). Then:

(i) Fuzzy compatible similarity relation $\alpha$ on $\mathrm{G}$ is called a fuzzy congruence.

(ii) Fuzzy equivalence relation $\alpha$ on $G$ is called a fuzzy congruence if

$$
\alpha(x * t, y * z) \geq \alpha(x, y) \wedge \alpha(t, z) .
$$


Proposition 2.5. [3] Let $P$ and $Q$ be fuzzy congruences on semigroup $S$. Then:

(i) If $P \circ Q=Q \circ P$, then $P \circ Q$ is a fuzzy congruence on $S$.

(ii) If $P \circ Q$ is fuzzy congruence on $S$, then $P \circ Q=P \vee Q$.

Proposition 2.6. [3] Let $P$ and $Q$ be fuzzy congruences on group $G$. Then:

(i) $P \circ Q=Q \circ P$.

(ii) $(C(G), \circ)$ is a semilattice, that $C(G)$ is the family of all fuzzy congruences of a group $G$.

(iii) $(C(G), \subseteq, \cap, \circ)$ is a modular lattice.

Lemma 2.7. [4] Let $\alpha$ be a fuzzy congruence on group $G$. Then:

(i) $\alpha(x * a * y, x * b * y)=\alpha(x * a, x * b)=\alpha(a * y, b * y)=\alpha(a, b)$.

(ii) $\alpha\left(a^{-1}, b^{-1}\right)=\alpha(a, b)$.

Here $x, y, z, a, b \in G$.

Proposition 2.8. [10] Let $\mu$ be a fuzzy ideal of semigroup $S$. Then the fuzzy relation $\theta_{\mu}$ as follows:

$$
\theta_{\mu}(x, y)=\left\{\begin{array}{lr}
1 & \text { if } x=y \\
\mu(x) \wedge \mu(y) & \text { if } x \neq y
\end{array}\right.
$$

is a fuzzy congruence on $S$.

\section{On Fuzzy Congruence on Groups}

Example 3.1. Let $G=Z_{4}$. Then fuzzy relation

$$
\alpha(x, y)= \begin{cases}1 & \text { if } x=y \\ .5 & \text { if }(x, y) \in\{(2,3),(3,2)\} \\ .3 & \text { otherwise }\end{cases}
$$

is a fuzzy equivalence relation on $G$, but is not a fuzzy congruence on $\mathrm{G}$.

Since $\alpha(2+2,3+2) \ngtr \alpha(2,3) \wedge \alpha(2,2)$.

Lemma 3.2. Let $\alpha$ be a fuzzy congruence on $G$. Then:

If $\alpha(x, y)<\alpha(z, t)$, then $\alpha(x, y)=\alpha(x * z, y * t)$.

Proof. Let $\alpha(x, y)<\alpha(z, t)$. It is clear that $\alpha(x, y) \leq \alpha(x * z, y * t)$. On the other hand by suppose we have $\alpha(x, y)=\alpha\left(x * z * z^{-1}, y * t * t^{-1}\right) \geq$ $\alpha\left(z^{-1}, t^{-1}\right) \wedge \alpha(x * z, y * t)$. Hence $\alpha(x, y) \geq \alpha(x * z, y * t)$. 
Theorem 3.3. Let $H$ be a normal subgroup of $G$. Then

$$
\alpha(x, y)=\left\{\begin{array}{cc}
1 & \text { if } x * y^{-1} \in H \\
t & \text { otherwise }
\end{array}\right.
$$

where $t \in[0,1)$, is a fuzzy congruence on $G$.

Proof. It is clear that $\alpha$ is a fuzzy reflexive and symmetric. To transitivity it is sufficient to prove, if $\alpha(x, t)=\alpha(t, y)=1$, then $\alpha(x, y)=1$. Therefore $x * t^{-1} \in H$ and $t * y^{-1} \in H$ so we have $x * t^{-1} * t * y^{-1}=x * y^{-1} \in H$. Now we prove $\alpha$ is compatible, if $\alpha(x, y)=\alpha(t, z)=1$, then $x * y^{-1} \in H$ and $t * z^{-1} \in H$. Since $H \triangleleft G$, so $y * t * z^{-1} * y^{-1} \in H$. Hence

$$
x * y^{-1} * y * t * z^{-1} * y^{-1}=x * t * z^{-1} * y^{-1}=x * t *(y * z)^{-1} \in H
$$

So $\alpha(x * t, y * z)=1$. This completes the proof.

Definition 3.4. Let $(G, *)$ be a semigroup and $\alpha$ and $\beta$ be fuzzy relations on $\mathrm{G}$. Then the composition $\alpha \odot \beta$ is defined as follows:

$$
(\alpha \odot \beta)(x, y)=\bigvee_{\substack{x=a * c \\ y=b * d}}\{\alpha(a, b) \wedge \beta(c, d)\}
$$

It is clear that $\alpha \odot \beta \in F R(G)$. Since operation $*$ is associative and by definition 3.4, we have $\alpha \odot(\beta \odot \gamma)=(\alpha \odot \beta) \odot \gamma$ for all $\alpha, \beta, \gamma \in F R(G)$.

Theorem 3.5. Let $F R(G)$ be the family of all fuzzy relations on semigroup G. Then $(F R(G), \odot)$ is a semigroup.

Theorem 3.6. Let $F C(C G)$ be the family of all fuzzy congruences on commutative semigroup ( abelian group) $G$.Then $(F C(C G), \odot)$ is a semigroup.

Proof. Suppose that $\alpha$ and $\beta$ are fuzzy congruences on commutative semigroup G. Then by Theorem 3.5, it is sufficient to prove that $\alpha \odot \beta$ is a fuzzy congruence on $\mathrm{G}$.

$$
\begin{aligned}
(\alpha \odot \beta)(x, x) & =\bigvee_{\substack{x=a * c \\
x=b * d}}\{\alpha(a, b) \wedge \beta(c, d)\} \\
& \geq \bigvee_{\substack{x=a * c \\
x=a * c}}\{\alpha(a, a) \wedge \beta(c, c)\} \\
& =1 \text { (reflexive) }
\end{aligned}
$$




$$
\begin{aligned}
& (\alpha \odot \beta)(x, y)=\bigvee_{\substack{x=a * c \\
y=b * d}}\{\alpha(a, b) \wedge \beta(c, d)\} \\
& =\bigvee_{\substack{y=b * d \\
x=a * c}}\{\alpha(b, a) \wedge \beta(d, c)\} \\
& =(\alpha \odot \beta)(y, x) \quad(\text { symmetric }) \\
& (\alpha \odot \alpha)(x, y)=\bigvee_{\substack{x=a * c \\
y=b * d}}\{\alpha(a, b) \wedge \alpha(c, d)\}=\bigvee_{\substack{x=a * c \\
y=b * d}}\{\alpha(a * c, b * c) \wedge \alpha(b * c, b * d)\} \\
& \leq \bigvee_{\substack{x=a * c \\
y=b * d}}\{\alpha(a * c, b * d)\} \\
& =\alpha(x, y)
\end{aligned}
$$

Therefore $\alpha \odot \alpha \leq \alpha$.

$$
\begin{aligned}
(\alpha \odot \beta)(x, y) & =\bigvee_{\substack{x=a * c \\
y=b * d}}\{\alpha(a, b) \wedge \beta(c, d)\} \\
& =\bigvee_{\substack{x=c * a \\
y=d * b}}\{\beta(c, d) \wedge \alpha(a, b)\} \\
& =(\beta \odot \alpha)(x, y)
\end{aligned}
$$

By associative and commutative property of operation $\odot$ we have

$$
(\alpha \odot \beta) \odot(\alpha \odot \beta)=(\alpha \odot \alpha) \odot(\beta \odot \beta) \leq \alpha \odot \beta
$$

So $\alpha \odot \beta$ is transitive.

For $x, y, t, z \in G$ we consider elements of $\mathrm{G}$ such that $x=a_{1} * c_{1}, y=b_{1} * d_{1}$, $t=a_{2} * c_{2}$ and $z=b_{2} * d_{2}$. Therefore

$$
\begin{aligned}
& (\alpha \odot \beta)(x * t, y * z)=\bigvee_{\substack{x * t=a * c \\
y * z=b * d}}\{\alpha(a, b) \wedge \beta(c, d)\} \\
& =\bigvee_{\substack{x * t=\left(a_{1} * c_{1}\right) *\left(a_{2} * c_{2}\right) \\
y * z=\left(b_{1} * d_{1}\right) *\left(b_{2} * d_{2}\right)}}\left\{\alpha\left(a_{1} * a_{2}, b_{1} * b_{2}\right) \wedge \beta\left(\left(c_{1} * c_{2}, d_{1} * d_{2}\right)\right\}\right. \\
& =\bigvee_{\substack{x * t=\left(a_{1} * a_{2}\right) *\left(c_{1} * c_{2}\right) \\
y * z=\left(b_{1} * b_{2}\right) *\left(d_{1} * d_{2}\right)}}\left\{\alpha\left(a_{1} * a_{2}, b_{1} * b_{2}\right) \wedge \beta\left(\left(c_{1} * c_{2}, d_{1} * d_{2}\right)\right\}\right.
\end{aligned}
$$




$$
\begin{aligned}
& \geq \bigvee_{\substack{x * t=\left(a_{1} * a_{2}\right) *\left(c_{1} * c_{2}\right) \\
y * z=\left(b_{1} * b_{2}\right) *\left(d_{1} * d_{2}\right)}}\left\{\alpha ( a _ { 1 } , b _ { 1 } ) \wedge \beta \left(\left(c_{1}, d_{1}\right) \wedge \alpha\left(a_{2}, b_{2}\right) \wedge \beta\left(\left(c_{2}, d_{2}\right)\right\}\right.\right. \\
& =\bigvee_{\substack{x=a_{1} * c_{1} \\
y=b_{1} * d_{1}}}\left\{\alpha ( a _ { 1 } , b _ { 1 } ) \wedge \beta ( ( c _ { 1 } , d _ { 1 } ) \} \wedge \bigvee _ { \substack { t = a _ { 2 } * c _ { 2 } \\
z = b _ { 2 } * d _ { 2 } } } \left\{\alpha\left(a_{2}, b_{2}\right) \wedge \beta\left(\left(c_{2}, d_{2}\right)\right\}\right.\right. \\
& =(\alpha \odot \beta)(x, y) \wedge(\alpha \odot \beta)(t, z)
\end{aligned}
$$

Definition 3.7. Let $A, B, C$ and $D$ be subsets of semigroup $S, \alpha$ be a fuzzy relation on $S$ and $f$ and $g$ be morphisms from $A$ to $B$ and $C$ to $D$ respectively . Then:

(i) $\alpha$ is called $f \times g$-invariant if $f \times g(x, y)=f \times g(z, t) \Rightarrow \alpha(x, y)=\alpha(z, t)$.

(ii) $\mathrm{f}$ and $\mathrm{g}$ are called $\mathrm{f}$ - $\mathrm{g}$ one to one if $f(x)=g(y) \Longrightarrow x=y$.

Theorem 3.8. Let $f$ and $g$ be homomorphisms from $G$ onto $H, G$ and $H$ be semigroups (groups), $f$ and $g$ be $f$ - $g$ one to one and $\alpha$ be a $f \times g$-invariant fuzzy congruence on $G$. Then

$$
(f \times g)(\alpha)(x, y)= \begin{cases}\bigvee \alpha(a, b) & \text { if }(a, b) \in(f \times g)^{-1}(x, y) \\ 0 & \text { otherwise }\end{cases}
$$

is a fuzzy congruence on $H$.

Proof. For $x \in H$

$$
\begin{aligned}
(f \times g)(\alpha)(x, x) & =\underset{\substack{\left(a, a^{\prime}\right) \in(f \times g)^{-1}(x, x)\\
}}{ } \alpha\left(a, a^{\prime}\right) \\
& \bigvee_{\substack{f(a)=x \\
g\left(a^{\prime}\right)=x}} \alpha\left(a, a^{\prime}\right) \\
= & \alpha(a, a)=1
\end{aligned}
$$

If $x, y \in H$

$$
\begin{aligned}
(f \times g)(\alpha) \circ(f \times g)(\alpha)(x, y)= & \bigvee_{z \in H}\left\{\left(\underset{(a, b) \in(f \times g)^{-1}(x, z)}{\bigvee^{\prime}} \alpha(a, b)\right)\right. \\
& \left.\wedge\left(\bigvee_{\left(b^{\prime}, c\right) \in(f \times g)^{-1}(z, y)} \alpha\left(b^{\prime}, c\right)\right)\right\}
\end{aligned}
$$




$$
\begin{aligned}
& =\bigvee_{z \in H}\left\{\left(\bigvee_{\substack{f(a)=x \\
g(b)=z}} \alpha(a, b)\right) \wedge\left(\bigvee_{\substack{f\left(b^{\prime}\right)=z \\
g(c)=y}} \alpha\left(b^{\prime}, c\right)\right)\right\} \\
& \left.=\bigvee_{z \in H} \bigvee_{\substack{f(a)=x \\
g(b)=f\left(b^{\prime}\right)=z \\
g(c)=y}}(\alpha(a, b)) \wedge \alpha\left(b^{\prime}, c\right)\right) \\
& \left.=\bigvee_{z \in H} \bigvee_{\substack{f(a)=x \\
f(b)=g(b)=z \\
g(c)=y}}(\alpha(a, b)) \wedge \alpha(b, c)\right) \\
& \left.\leq \bigvee_{\substack{f(a)=x \\
f(b)=g(b)=z \\
g(c)=y}} \bigvee_{b \in G}(\alpha(a, b)) \wedge \alpha(b, c)\right) \\
& =\bigvee_{\substack{f(a)=x \\
g(c)=y}} \alpha(a, c) \\
& =\bigvee_{(a, c) \in(f \times g)^{-1}(x, y)} \alpha(a, c)=f \times g(\alpha)(x, y)
\end{aligned}
$$

By the definition it is clear that $f \times g(\alpha)$ is symmetric. If $x, y, z, t \in H$, then

$$
\begin{aligned}
& (f \times g)(\alpha)(x * z, y * t)=\bigvee_{(a, b) \in(f \times g)^{-1}(x * z, y * t)} \alpha(a, b) \\
& =\bigvee_{\substack{f(a)=x * z \\
g(b)=y * t}} \alpha(a, b) \\
& =\begin{array}{l}
\bigvee_{u \in f^{-1}(x), v \in f^{-1}(z)} \alpha(a, b) \\
f(a)=f(u * v) \\
g(b)=g(w * s) \\
w \in g^{-1}(y), s \in g^{-1}(t)
\end{array} \\
& =\bigvee_{\substack{u \in f^{-1}(x), w \in g^{-1}(y) \\
v \in f^{-1}(z), s \in g^{-1}(t)}} \alpha(u * v, w * s) \\
& \geq \quad \quad \quad \alpha(u, w) \wedge \alpha(v, s) \\
& u \in f^{-1}(x), w \in g^{-1}(y) \\
& v \in f^{-1}(z), s \in g^{-1}(t) \\
& =\bigvee_{\substack{u \in f^{-1}(x) \\
w \in g^{-1}(y)}} \alpha(u, w) \wedge \bigvee_{\substack{v \in f^{-1}(z) \\
s \in g^{-1}(t)}} \alpha(v, s)
\end{aligned}
$$




$$
=(f \times g)(\alpha)(x, y) \wedge(f \times g)(\alpha)(z, t) .
$$

Corollary 3.9. (i) If $f$ and $g$ are isomorphisms from $G$ onto $H, f$ and $g$ are $f$ - $g$ one to one and $\alpha$ is a fuzzy congruence on $G$, then $(f \times g)(\alpha)$ is a fuzzy congruence on $H$.

(ii) If $f$ is a isomorphism from $G$ onto $H$ and $\alpha$ is a fuzzy congruence on $G$, then $(f \times f)(\alpha)$ is a fuzzy congruence on $H$.

Theorem 3.10. Let $f$ be a homomorphism from $G$ into $H$ and $\theta$ be a fuzzy congruence on $H$. Then

$$
(f \times f)^{-1}(\theta)(x, y)=\theta(f \times f(x, y))=\theta(f(x), f(y))
$$

is a fuzzy congruence on $G$.

Proof. The proof is straightforward.

Theorem 3.11. Let $f$ be an isomorphism from $G$ onto $H$ and $\alpha$ and $\theta$ be fuzzy congruences on $G$ and $H$ respectively. Then $(f \times f)\left((f \times f)^{-1}(\theta)\right)=\theta$ and $(f \times f)^{-1}((f \times f)(\alpha))=\alpha$.

Proof. By Corollary 3.9 and Theorem 3.10, the proof is easy.

\section{Fuzzy Normal Congruences}

Definition 4.1. Let $\alpha$ be a fuzzy congruence on semigroup $G$. Then $\alpha$ is called a fuzzy normal congruence on $G$, if

$$
\alpha(x * t, y * z)=\alpha(t * x, z * y) \text { for all } x, y, t, z \in G
$$

Lemma 4.2. Let $\alpha$ be a fuzzy congruence on $G$ and $x, y, z, t \in G$. Then:

(i) $\alpha$ is a fuzzy normal congruence on $G$, if and only if $\alpha\left(x^{-1} * t * x, y^{-1} *\right.$ $z * y)=\alpha(t, z)$.

(ii) If $G$ is an abelian group, then $\alpha$ is a fuzzy normal congruence on $G$.

(iii) If $\mu$ is a fuzzy normal subgroup of $G$, then $\alpha_{\mu}$ is a fuzzy normal congruence on $G$. 
Proof. Suppose that $\alpha$ is a fuzzy normal congruence on $\mathrm{G}$ and $x, y, z, t \in G$. Then

$$
\alpha\left(x^{-1} * t * x, y^{-1} * z * y\right)=\alpha\left(t * x^{-1} * x, z * y^{-1} * y\right)=\alpha(t, z)
$$

Conversely

$$
\alpha(t * x, z * y)=\alpha\left(x^{-1} *(x * t) * x, z^{-1} *(z * y) * z\right)=\alpha(x * t, y * z)=
$$

The proofs of (ii), (iii) are trivial.

Example 4.3. Let $G=\{a, b, c, d, e, f\}$, that $a=(1), b=(12)(36)(45)$, $c=(13)(25)(46), d=(14)(26)(35), e=(156)(234)$ and $f=(165)(243)$. Then

$$
\mu(x)= \begin{cases}1 & \text { if } x=a \\ .5 & \text { if } x=c, d, e, f \\ .3 & \text { if } x=b\end{cases}
$$

is a fuzzy subgroup of $\mathrm{G}$. By Proposition $2.8, \theta_{\mu}$ is a fuzzy congruence on $\mathrm{G}$. But it is not fuzzy normal congruence on G. since

$$
\begin{aligned}
\theta_{\mu}(a * f, c * e)=\mu(a * f) \wedge \mu(c * e) & =\mu(a) \wedge \mu(d)=.5 \\
& \neq \mu(a) \wedge \mu(b) \\
& =\mu(f * a) \wedge \mu(e * c) \\
& =\theta_{\mu}(f * a, e * c)
\end{aligned}
$$

Suppose that

$$
\mu(x)= \begin{cases}1 & \text { if } x=a \\ .5 & \text { if } x=e, f \\ .3 & \text { if } x=b, c, d\end{cases}
$$

Then it is easy to verify that $\theta_{\mu}$ is a fuzzy normal congruence on $\mathrm{G}$.

In the following theorem we suppose that in (i) homomrophisms $\mathrm{f}$ and $\mathrm{g}$ satisfy in the conditions of Theorems 3.8 and in (i) $\mathrm{f}$ is a homomorphism.

Theorem 4.4. Let $\alpha$ and $\beta$ be fuzzy normal congruences on $G$ and $H$ respectively. Then:

(i) $(f \times g)(\alpha)$ is a fuzzy normal congruence on $H$.

(ii) $(f \times f)^{-1}(\beta)$ is a fuzzy normal congruence on $G$. 
Proof. (i)

$$
\begin{aligned}
& (f \times g)(\alpha)(x * z, y * t)=\bigvee_{(a, b) \in(f \times g)^{-1}(x * z, y * t)} \alpha(a, b) \\
& =\bigvee_{\substack{f(a)=x * z \\
g(b)=y * t}} \alpha(a, b) \\
& =\bigvee_{f(a)=f(u * v)} \alpha(a, b) \\
& g(b)=g(w * s) \quad w \in g^{-1}(y), s \in g^{-1}(t) \\
& =\bigvee_{\substack{u \in f^{-1}(x), w \in g^{-1}(y) \\
v \in f^{-1}(z), s \in g^{-1}(t)}} \alpha(u * v, w * s) \\
& =\bigvee_{\substack{u \in f^{-1}(x), w \in g^{-1}(y) \\
v \in f^{-1}(z), s \in g^{-1}(t)}} \alpha(v * u, s * w) \\
& =\quad \bigvee \quad \alpha\left(a^{\prime}, b^{\prime}\right) \\
& f\left(a^{\prime}\right)=f(v * u) \quad u \in f^{-1}(x), v \in f^{-1}(z) \\
& g\left(b^{\prime}\right)=g(s * w) \quad w \in g^{-1}(y), s \in g^{-1}(t) \\
& =\quad \vee \quad \alpha\left(a^{\prime}, b^{\prime}\right) \\
& f\left(a^{\prime}\right)=z * x \quad u \in f^{-1}(x), v \in f^{-1}(z) \\
& g\left(b^{\prime}\right)=t * y \quad w \in g^{-1}(y), s \in g^{-1}(t) \\
& =\quad \quad \alpha\left(a^{\prime}, b^{\prime}\right) \\
& \left(a^{\prime}, b^{\prime}\right) \in(f \times g)^{-1}(z * x, t * y) \\
& =(f \times g)(\alpha)(z * x, t * y)
\end{aligned}
$$

(ii) The proof is obvious.

\section{Fuzzy Coset Relations}

Definition 5.1. Let $\alpha$ be a fuzzy congruence on G. Then for $a, b \in G$, the fuzzy relation $\alpha^{(a, b)}$ on $\mathrm{G}$ defined by $\alpha^{(a, b)}(x, y)=\alpha\left(a * x^{-1}, b * y^{-1}\right)$, for all $x, y \in G$, is called a fuzzy coset relation determined by $a, b$ and $\alpha$. 
Lemma 5.2. Let $\alpha$ be a fuzzy normal congruence, $\alpha^{(a, b)}$ and $\alpha^{(c, d)}$ be fuzzy coset relations on $G$. Then

$$
\alpha^{(a, b)}=\alpha^{(c, d)} \text { if and only if } \alpha\left(a * c^{-1}, b * d^{-1}\right)=1
$$
$\left.d^{-1}\right)$.

Proof. Suppose that $\alpha\left(a * c^{-1}, b * d^{-1}\right)=1$. Then $\alpha^{(a, b)}(x, y) \leq \alpha\left(a * c^{-1}, b *\right.$ If $\alpha^{(a, b)}(x, y)=\alpha\left(a * c^{-1}, b * d^{-1}\right)$, then

$$
\begin{aligned}
\alpha\left(c * x^{-1}, d * y^{-1}\right) & =\alpha\left(a^{-1} *\left(c * x^{-1}\right) * a, b^{-1} *\left(d * y^{-1}\right) * b\right) \\
& =\alpha\left(\left(a^{-1} * c\right) *\left(x^{-1} * a\right),\left(b^{-1} * d\right) *\left(y^{-1} * b\right)\right) \\
& \geq \alpha\left(a^{-1} * c, b^{-1} * d\right) \wedge \alpha\left(x^{-1} * a, y^{-1} * b\right) \\
& =\alpha\left(a * x^{-1}, b * y^{-1}\right) \wedge \alpha\left(a * c^{-1}, b * d^{-1}\right) \\
& =\alpha\left(a * x^{-1}, b * y^{-1}\right)
\end{aligned}
$$

By the similar way we have $\alpha^{(a, b)}(x, y) \leq \alpha^{(c, d)}(x, y)$. Thus $\alpha^{(a, b)}(x, y)=$ $\alpha^{(c, d)}(x, y)$.

Now if $\alpha^{(a, b)}(x, y)<\alpha\left(a * c^{-1}, b * d^{-1}\right)$, then by Lemm 3.2, we have

$$
\begin{aligned}
\alpha\left(a * x^{-1}, b * y^{-1}\right) & =\alpha\left(\left(a * x^{-1}\right) *\left(a * c^{-1}\right)^{-1},\left(b * y^{-1}\right) *\left(b * d^{-1}\right)^{-1}\right) \\
& =\alpha\left(\left(a * x^{-1}\right) *\left(c * a^{-1}\right),\left(b * y^{-1}\right) *\left(d * b^{-1}\right)\right) \\
& =\alpha\left(a *\left(x^{-1} * c\right) * a^{-1}, b *\left(y^{-1} * d\right) * b^{-1}\right) \\
& =\alpha\left(x^{-1} * c, y^{-1} * d\right) \\
& =\alpha\left(c * x^{-1}, d * y^{-1}\right) .
\end{aligned}
$$

Definition 5.3. If $\alpha^{(a, b)}$ and $\alpha^{(c, d)}$ are fuzzy coset relations, then the composition $\alpha^{(a, b)} \odot \alpha^{(c, d)}$ is defined as follows:

$$
\alpha^{(a, b)} \odot \alpha^{(c, d)} \quad(x, y)=\bigvee_{\substack{x=x_{1} * x_{2} \\ y=y_{1} * y_{2}}}\left\{\alpha^{(a, b)}\left(x_{1}, y_{1}\right) \wedge \alpha^{(c, d)}\left(x_{2}, y_{2}\right)\right\}
$$

Proposition 5.4. Let $\alpha$ be a fuzzy normal congruence on $G, \alpha^{(a, b)}$ and $\alpha^{(c, d)}$ be fuzzy coset relations on $G$. Then

$$
\alpha^{(a, b)} \odot \alpha^{(c, d)}(x, y)=\alpha^{(a * c, b * d)}(x, y) .
$$

Proof. First, we prove the operation $\odot$ is well-defined. Suppose that $\alpha^{(a, b)}=$ $\alpha^{(c, d)}$ and $\alpha^{(e, f)}=\alpha^{(g, h)}$. Then

$$
\alpha\left((a * e) *(c * g)^{-1},(b * f) *(d * h)^{-1}\right)
$$




$$
\begin{aligned}
& \geq \alpha\left((a * e) *\left(g^{-1} * c^{-1}\right),(b * f) *\left(h^{-1} * d^{-1}\right)\right) \\
& =\alpha\left(a *\left(e *\left(g^{-1} * c^{-1}\right)\right), b *\left(f *\left(h^{-1} * d^{-1}\right)\right)\right) \\
& =\alpha\left(\left(e *\left(g^{-1} * c^{-1}\right)\right) * a,\left(f *\left(h^{-1} * d^{-1}\right)\right) * b\right) \\
& =\alpha\left(\left(e * g^{-1}\right) *\left(c^{-1} * a\right),\left(f * h^{-1}\right) *\left(d^{-1} * b\right)\right) \\
& =\alpha\left(e * g^{-1}, f * h^{-1}\right) \wedge \alpha\left(a * c^{-1}, b * d^{-1}\right)=1
\end{aligned}
$$

Therefore $\alpha^{(a, b)} \odot \alpha^{(e, f)}=\alpha^{(c, d)} \odot \alpha^{(g, h)}$.

For all $x_{1}, x_{2}, y_{1}, y_{2} \in V$ such that $x=x_{1} * x_{2}$ and $y=y_{1} * y_{2}$ we have

$$
\begin{aligned}
\alpha^{(a * c, b * d)}(x, y) & =\alpha\left((a * c) *\left(x_{2}^{-1} * x_{1}^{-1}\right),(b * d) *\left(y_{2}^{-1} * y_{1}^{-1}\right)\right. \\
& =\alpha\left(a *\left(c * x_{2}^{-1} * x_{1}^{-1}\right), b *\left(d * y_{2}^{-1} * y_{1}^{-1}\right)\right) \\
& =\alpha\left(\left(c * x_{2}^{-1} * x_{1}^{-1}\right) * a,\left(d * y_{2}^{-1} * y_{1}^{-1}\right) * b\right) \\
& =\alpha\left(\left(c * x_{2}^{-1}\right) *\left(x_{1}^{-1} * a\right),\left(d * y_{2}^{-1}\right) *\left(y_{1}^{-1} * b\right)\right) \\
& \geq \alpha\left(c * x_{2}^{-1}, d * y_{2}^{-1}\right) \wedge \alpha\left(x_{1}^{-1} * a, y_{1}^{-1} * b\right) \\
& =\alpha\left(c * x_{2}^{-1}, d * y_{2}^{-1}\right) \wedge \alpha\left(a * x_{1}^{-1}, b * y_{1}^{-1}\right) \\
& =\alpha^{(a, b)}\left(x_{1}, y_{1}\right) \wedge \alpha^{(c, d)}\left(x_{2}, y_{2}\right)
\end{aligned}
$$

Therefore

$$
\alpha^{(a * c, b * d)}(x, y) \geq \bigvee_{\substack{x=x_{1} * x_{2} \\ y=y_{1} * y_{2}}}\left\{\alpha^{(a, b)}\left(x_{1}, y_{1}\right) \wedge \alpha^{(c, d)}\left(x_{2}, y_{2}\right)\right\}=\alpha^{(a, b)} \odot \alpha^{(c, d)}(x, y)
$$

Conversely

$$
\begin{aligned}
\alpha^{(a, b)} \odot \alpha^{(c, d)}(x, y) & =\bigvee_{\substack{x=x_{1} * x_{2} \\
y=y_{1} * y_{2}}}\left\{\alpha^{(a, b)}\left(x_{1}, y_{1}\right) \wedge \alpha^{(c, d)}\left(x_{2}, y_{2}\right)\right\} \\
& \geq \bigvee_{\substack{x=x_{1} * x_{2} \\
y=y_{1} * y_{2}}}\left\{\alpha^{(a, b)}\left(x_{1}, y_{1}\right) \wedge \alpha^{(c, d)}\left(x_{2}, y_{2}\right)\right\} \\
& =\bigvee_{\substack{x=x_{1} * x_{2} \\
y=y_{1} * y_{2}}}\left\{\alpha\left((a * c) *\left(x_{1} * x_{2}\right)^{-1},(b * d) *\left(y_{1} * y_{2}\right)^{-1}\right)\right. \\
& =\alpha^{(a * c, b * d)}(x, y) .
\end{aligned}
$$

Theorem 5.5. Let $\alpha$ be a fuzzy normal congruence on $G$. Then $((G \times$ $\left.G)_{\alpha}, \odot\right)$ is a group, where $(G \times G)_{\alpha}=\left\{\alpha^{(a, b)} \mid a, b \in G\right\}$. 
Proof. Since $\mathrm{G}$ is a group and by Proposition 5.4, $(G \times G)_{\alpha}$ is closed under the operation $\odot$.

$$
\begin{aligned}
{\left[\left(\alpha^{(a, b)} \odot \alpha^{(c, d)}\right) \odot \alpha^{(e, f)}\right](x, y) } & =\alpha^{(a * c, b * d)} \odot \alpha^{(e, f)}(x, y) \\
& =\alpha^{((a * c) * e,(b * d) * f)}(x, y) \\
& =\alpha^{(a *(c * e), b *(d * f))}(x, y) \\
& =\alpha^{(a, b)} \odot \alpha^{(c * e, d * f)}(x, y) \\
& =\left[\alpha^{(a, b)} \odot\left(\alpha^{(c, d)} \odot \alpha^{(e, f)}\right)\right](x, y) .
\end{aligned}
$$

Thus the operation $\odot$ is associative. $\alpha^{(e, e)}$ is identity element, and $\alpha^{\left(a^{-1}, b^{-1}\right)}$ is inverse of $\alpha^{(a, b)} \in(G \times G)_{\alpha}$. Therefore $(G \times G)_{\alpha}$ is a group.

Theorem 5.6. Let $\alpha$ be a fuzzy congruence on $G$. Then the $\operatorname{map} \varphi: G \times$ $G \longrightarrow(G \times G)_{\alpha}$ defined by $\varphi(a, b)=\alpha^{(a, b)}, \forall a, b \in G$ is a onto homomorphism with:

(i) $\varphi(a * c, b * c)=\varphi(a, b) \odot \varphi(c, d)$.

(ii) $\operatorname{Ker} \varphi=I_{G}$.

Proof. Clearly, $\varphi$ is an onto homomorphism and

$$
\varphi(a * c, b * c)=\alpha^{(a * c, b * d)}=\alpha^{(a, b)} \odot \alpha^{(c, d)}=\varphi(a, b) \odot \varphi(c, d) .
$$

If $(x, y) \in \operatorname{Ker} \varphi$, then $\varphi(x, y)=\alpha^{(x, y)}=\alpha^{(e, e)}$ and so by lemma 5.2 , we have $\alpha(x, y)=1$. Therefor $(x, y) \in I_{G}$, which completes the proof.

Corollary 5.7. Let $\alpha$ be a fuzzy normal congruence on $G$. Then

$$
(G \times G) / I_{G} \cong(G \times G)_{\alpha} .
$$

\section{Conclusion}

We know the equivalence classes induced by a congruence relation to study an algebraic system has a vital role. For instance the theory of rough sets and approximation sets has close relationship with congruence relation and equivalence classes. Similarly fuzzy congruence relations have this role in study of fuzzy algebraic systems. In this work we defined the concepts of fuzzy normal congruences and fuzzy coset relations in semigroups and groups. In a natural way the level subset of a fuzzy normal congruence must be a normal congruence that so far has not defined and we tend to study this concept in other work. 


\section{Acknowledgments}

The author would like to thank the referees for their valuable suggestions and comments.

\section{References}

[1] S. Abou-Zaid, On fuzzy subgroups, Fuzzy Sets Systems, 55 (1988), 237-240, doi: 10.1016/0165-0114(93)90137-7.

[2] M.K. Chakraborty, M. Das, Reduction of fuzzy strict order relations, Fuzzy Sets and Systems, 15 (1985), 33-44, doi: 10.1016/0165-0114(85)90014-4.

[3] J.P. Kim, D.R. Bae, Fuzzy congruences in groups, Fuzzy Sets Systems, 85 (1997), 115120, doi: 10.1016/0165-0114(95)00334-7.

[4] N. Kuroki, Fuzzy congruences and fuzzy normal subgroups, Inform. Sci., 60 (1992) 247259, doi: 10.1016/0020-0255(92)90013-X.

[5] N.P. Mukherjee, Fuzzy normal subgroups and fuzzy cosets, Inform. Sci. 34 (1984), 225239, doi: 10.1016/0020-0255(84)90050-1.

[6] V. Murali, Fuzzy equivalence relations, Fuzzy Sets and Systems, 30, No. 2 (1989), 155163, doi: 10.1016/0165-0114(89)90077-8.

[7] A. Rosenfeld, Fuzzy Groups, Journal of Mathematical Analysis and Applications, 35 (1971), 512-517, doi: 10.1016/0022-247X(71)90199-5.

[8] M.A. Samhan, T.M.G. Ahsanullah, Fuzzy congruence on groups and rings, Int. J. Math. and Math. Sci., 17 (1994), 469-474, doi: 10.1155/S0161171294000682.

[9] M. Samhan, Fuzzy congruence in semigroups, Inform. Sci., 74 (1993), 165-175, doi: 10.1016/0020-0255(93)90132-6.

[10] X.-Y. Xie, Fuzzy Rees congruences on semigroups, Fuzzy Sets and Systems, 102 (1999), 353-359, doi: 10.1016/S0165-0114(97)00156-5.

[11] L.A. Zadeh, Fuzzy sets, Information and Control, 8 (1965), 338-353, doi: 10.1016/S00199958(65)90241-X. 